Bull. Austral. Math. Soc.

Vol. 53 (1996) [499-500]

\title{
ON THE CANCELLATION PROBLEM OF ZARISKI
}

\section{Dongho Byeon and Hyun Kwang Kim}

Let $K_{1}$ and $K_{2}$ be extension fields over a field $K$ with char $K=p>0$. Assume $L=K_{1}\left(x_{1}\right)=K_{2}\left(x_{2}\right) \supset K$ where $x_{i}$ is transcendental over $K_{i}$, for $i=1,2$. In this paper we prove that if $K_{1}$ is a perfect field, then $K_{1}=K_{2}$.

Let $K_{1}$ and $K_{2}$ be finitely generated extensions of a field $K$ and let $x_{i}$ be transcendental over $K_{i}, i=1,2$. The cancellation problem of Zariski [5] asks if $K_{1}\left(x_{1}\right)=K_{2}\left(x_{2}\right)$, must $K_{1}$ and $K_{2}$ be $K$-isomorphic? In general the answer is no [1]. However there are some special cases in which the answer is yes $[2,3,4,5]$. For example, it is known that the answer is yes if $\operatorname{char} K=0$ and $x_{1}=x_{2}[2,5]$. But for the case of a finite base field, very little is known. In this paper we shall prove the answer is yes for an important case of a finite base field, that is, if char $K=p>0$ and $K_{1}$ is a perfect field, then $K_{1} \cong K_{2}$. In this case we have a stronger result, namely $K_{1}=K_{2}$.

Theorem . Let $K_{1}$ and $K_{2}$ be extension fields over a field $K$ with char $K=$ $p>0$. Assume $L=K_{1}\left(x_{1}\right)=K_{2}\left(x_{2}\right) \supset K$ where $x_{i}$ is transcendental over $K_{i}$, for $i=1,2$. If $K_{1}$ is a perfect field, then $K_{1}=K_{2}$.

REMARK. In $[2,3,4,5]$, it is assumed that $K_{1}$ and $K_{2}$ are finitely generated extensions of $K$. However, in our Theorem this assumption in not required.

We start with a lemma.

LEMMA. Let $K_{1}$ and $K_{2}$ be fields as in the Theorem. If $K_{1}$ is a perfect field, then so is $K_{2}$.

ProOF: Let $\varphi$ be the Frobenius automorphism of $L$ so that $\varphi(a)=a^{p}$ for all $a \in L$, where $p=\operatorname{char} K>0$. Then $\varphi(L)=L^{p}=K_{1}^{p}\left(x_{1}^{p}\right)=K_{2}^{p}\left(x_{2}^{p}\right)$. Since $K_{1}^{p}=K_{1}$, $K_{1}\left(x_{1}^{p}\right)=K_{2}^{p}\left(x_{2}^{p}\right)$. Thus $\left[K_{2}\left(x_{1}\right): K_{2}^{p}\left(x_{2}^{p}\right)\right]=\left[K_{1}\left(x_{1}\right): K_{1}\left(x_{1}^{p}\right)\right]=p$. However $p=\left[K_{2}\left(x_{2}\right): K_{2}^{p}\left(x_{2}^{p}\right)\right]=\left[K_{2}\left(x_{2}\right): K_{2}\left(x_{2}^{p}\right)\right] \times\left[K_{2}\left(x_{2}^{p}\right): K_{2}^{p}\left(x_{2}^{p}\right)\right]=p \times\left[K_{2}\left(x_{2}^{p}\right): K_{2}^{p}\left(x_{2}^{p}\right)\right]$. So $\left[K_{2}\left(x_{2}^{p}\right): K_{2}^{p}\left(x_{2}^{p}\right)\right]=1$, that is, $K_{2}^{p}\left(x_{2}^{p}\right)=K_{2}\left(x_{2}^{p}\right)$. This implies that $K_{2}^{p}=K_{2}$.

Proof of TheOREM: Let $K_{1} K_{2}$ be the compositum of $K_{1}$ and $K_{2}$ in $L$. Then $L=K_{1} K_{2}\left(x_{1}, x_{2}\right)$ since $K_{1} K_{2}\left(x_{1}, x_{2}\right) \subset L$ and $L \subset K_{1} K_{2}\left(x_{1}, x_{2}\right)$ by the definition of

Received 8th August, 1995

Copyright Clearance Centre, Inc. Serial-fee code: 0004-9729/96 $\$ A 2.00+0.00$. 
compositum. First we show that $L$ is a transcendental extension over $K_{1} K_{2}$. By the Lemma $K_{2}$ is also a perfect field. So $L^{p^{n}}=K_{1}^{p^{n}}\left(x_{1}^{p^{n}}\right)=K_{2}^{p^{n}}\left(x_{2}^{p^{n}}\right)=K_{1}\left(x_{1}^{p^{n}}\right)=$ $K_{2}\left(x_{2}^{p^{n}}\right)$ for every positive integer $n$. Thus $K_{1} K_{2} \subset L^{p^{n}}$ for every positive integer $n$.

$$
\begin{aligned}
& L \quad=K_{1}\left(x_{1}\right)=K_{2}\left(x_{2}\right)=K_{1} K_{2}\left(x_{1}, x_{2}\right) \\
& L^{p}=K_{1}\left(x_{1}^{p}\right)=K_{2}\left(X_{2}^{p}\right) \\
& L^{p^{n}}=K_{1}\left(x_{1}^{p^{n}}\right)=K_{2}\left(x_{2}^{p^{n}}\right) \\
& K_{1} K_{2} \\
& \begin{array}{ll}
/ & \backslash \\
K_{1} & K_{2}
\end{array} \\
& 1_{K} 1
\end{aligned}
$$

But $\left[L: L^{p^{n}}\right]=p^{n}$ for every positive integer $n$. So $L$ is an infinite dimensional extension over $K_{1} K_{2}$. Since $L=K_{1} K_{2}\left(x_{1}, x_{2}\right), L$ must be a transcendental extension over $K_{1} K_{2}$. Now we claim that $K_{1} K_{2}$ must be algebraic over $K_{i}$, for $i=1,2$. Otherwise $1=t r . d_{K_{i}} K_{i}\left(x_{i}\right)=\operatorname{tr} . d_{K_{i}} K_{1} K_{2}+t r . d_{K_{1} K_{2}} L \geqslant 2$, for $i=1,2$. Since $K_{i}$ is algebraically closed in $L$, for $i=1,2$, we conclude that $K_{1} K_{2} \subset K_{i}$, for $i=1,2$. Hence $K_{1} K_{2}=K_{1}=K_{2}$.

\section{REFERENCES}

[1] A. Beauville, J.L. Colliot-Thélène, J.J. Sansuc and Sir P. Swinnerton-Dyer, 'Variétés stablement rationnelles non rationelles', Ann. of Math. 121 (1986), 283-315.

[2] J. Deveney, 'Automorphism groups of ruled function fields and a problem of Zariski', Proc. Amer. Math. Soc. 90 (1984), 178-180.

[3] J. Deveney, 'The cancellation problem for function fields', Proc. Amer. Math. Soc. 103 (1988), 363-364.

[4] M. Kang, 'A note on the birational cancellation problem', J. Pure Appl. Algebra 77 (1992), 141-154.

[5] M. Nagata, 'A Theorem on valuation rings and its applications', Nagoya Math. J. 29 (1967), 85-91.

Department of Mathematics

Pohang University of Science and Technology

Pohang Korea

e-mail: faust@posmath.postech.ac.kr 